Trauma Berufskrankh 2017· 19 (Suppl 3):S272-S274 https://doi.org/10.1007/s10039-017-0303-5 Online publiziert: 14. September 2017

๑) Springer Medizin Verlag GmbH 2017

CrossMark

\section{G. Matziolis}

Campus Eisenberg, Orthopädische Klinik des Universitätsklinikums Jena, Eisenberg, Deutschland

\section{Periprothetische Gelenksteife}

\section{Ursachen und Vorgehen}

Es existiert bislang keine allgemein akzeptierte Definition einer periprothetischen Gelenksteife. Am weitesten verbreitet ist die Annahme eines relevanten Streckdefizits $\left(>10^{\circ}\right)$ sowie einer Beugehemmung $\left(<90^{\circ}\right)[3,5,9,17]$. Diese Zahlenwerte spiegeln die praktische Relevanz für den betroffenen Patienten wider, da ab einem Streckdefizit von $10^{\circ}$ das Gehen unökonomisch wird und der patellofemorale Anpressdruck aufgrund des ständig aktivierten Streckapparates erhöht ist $[22,23]$. Eine Beugefähigkeit von weniger als $90^{\circ}$ erschwert das Aufstehen aus dem Sitzen sowie das Fahrradfahren ohne Hilfsmittel.

Das Risiko, eine periprothetische Gelenksteife zu entwickeln, ist nach Versorgung posttraumatischer Arthrosen mit ca. $25 \%$ signifikant höher als mit weniger als $10 \%$ nach primären Arthrosen $[14,30]$. Die Ursachen dafür sind vielfältig und lassen sich auch bei sorgfältiger Operationstechnik nicht vollends vermeiden, sodass Patienten vor endoprothetischem Ersatz bei posttraumatischer Gonarthrose explizit darüber aufgeklärt werden sollten.

\section{Ursachen}

Es können 3 Ursachenkomplexe für eine periprothetische Gelenksteife unterschieden werden: biologische, mechanische und gelenkferne Risikofaktoren. Es handelt sich dabei um Risikofaktoren und selten um kausale Ursachen, da ihr Vorliegen nicht zwangsläufig zu einer Gelenksteife führt und ihre Beseitigung nicht in jedem Fall die Gelenkbeweglichkeit verbessert. In der Patientenführung sollte daher von wahrscheinlichen Ursachen und einer möglichen Besserung der Beweglichkeit ausgegangen werden.

\section{Biologische Ursachen}

Eine Infektion mit Biofilm bildenden Erregern sollte im diagnostischen Algorithmus stets ausgeschlossen werden, auch wenn augenscheinlich andere Ursachen für eine Gelenksteife vorliegen. Die Basisdiagnostik stützt sich auf die Gelenkpunktion mit mikrobiologischer Untersuchung unter Langzeitbebrütung (14 Tage) [24] sowie Zellzahlbestimmung und -differenzierung [12, 28]. Darüber hinaus können die Leukozytenesterase, das C-reaktive Protein (CRP) und das $a$-Defensin bestimmt werden, wenngleich die Wertigkeit dieser Parameter noch nicht abschließend durch Metaanalysen gesichert ist [25]. Bei klinischem Verdacht auf eine Infektion und negativem Ergebnis der aus dem Punktat bestimmten Parameter können eine Analyse von periimplantären Schleimhaut- und Gewebeproben (empfohlen 5 Stück) und ein Inlaywechsel zur Sonikation durchgeführt werden [6, $15,26]$. Neben den Gewebeproben zur mikrobiologischen Analyse mit Langzeitbebrütung sollten dann auch Proben zur histologischen Untersuchung zur Klassifikation nach Morawietz und Krenn entnommen werden [18].

Es ist immer noch kontrovers, ob eine Allergie gegen Metalle (meist Nickel, Kobalt, Chrom), Knochenzement oder Benzoylperoxid eine periprothetische Gelenksteife verursachen kann. Zur Diagnostik werden klassische Hauttests sowie der Lymphozytentransformationstest (LTT) durchgeführt, wobei die diagnostische Wertigkeit beider Verfahren in Bezug auf implantatassoziierte Allergien nicht abschließend geklärt ist [19, 27].

Die primäre Arthrofibrose ist eine Ausschlussdiagnose, da es kein ak- zeptiertes beweisendes diagnostisches Kriterium gibt. Die Koinzidenz von übermäßiger intraartikulärer Bindegewebswucherung, Kapselverdickung und Bewegungseinschränkung weist auf eine primäre Arthrofibrose hin.

\section{Mechanische Ursachen}

Bei den mechanischen Risikofaktoren kann es sowohl durch ein direktes mechanisches Hindernis als auch indirekt über mechanisch bedingte Schmerzprovokation zu einer Bewegungseinschränkung kommen.

Die Diagnostik beginnt mit der klinischen Untersuchung und Prüfung der Seitenbandstabilität in bestmöglicher Streckung, bei $30^{\circ}, 60^{\circ}$ und - wenn erreichbar $-90^{\circ}$ Flexion. Bei Verdacht auf eine Instabilität sollte diese bildgebend durch gehaltene Aufnahmen verifiziert werden. Schmerzen, die provozierbar und lokalisiert sind, deuten auf eine mechanische Genese hin.

An dieklinische Untersuchung schließt sich die nativradiologische Bildgebung mit Ganzbeinaufnahmen, mindestens jedoch langen Aufnahmen in 2 Ebenen sowie mindestens einer tangentialen Patellaaufnahme („sunrise view“ oder „défile“() an. Malalignment der Komponenten in der Frontalebene scheint dabei weniger kritisch als in der Sagittal- oder Transversalebene für die Entwicklung einer Gelenksteife zu sein $[4,11,20]$.

Die Bildgebung wird durch eine Torsionscomputertomographie mit Abbildung des Schenkelhalses, der Malleolengabel und des gesamten Kniegelenkes ergänzt. Hier lassen sich Rotationsfehler von Femur- und Tibiakomponente, der Abstand zwischen Tuberositas tibiae und der Trochlea (TTTG) sowie Implantatüberhänge erkennen. 
Ein zu straffer Beuge- oder Streckspalt kann ebenfalls zur Gelenksteife führen, lässt sich aber weder bildgebend noch klinisch direkt nachweisen [10]. Liegen präoperative Bilder vor, lässt sich durch Vergleich mit den postoperativen Bildern abschätzen, ob der Streck- oder Beugespalt überspannt wurde. Ein etwaiges intraoperativ durchgeführtes Weichteilrelease bleibt dabei jedoch unberücksichtigt und kann allenfalls dem Operationsbericht entnommen werden.

Besonders eine Überspannung des anterioren Gelenkkompartiments sollte geprüft werden. Dazu wird im streng seitlichen Bild bei Flexion der Winkel zwischen Patellarband und anteriorer Tibiakortikalis (Patella-Tendon-Angle [PTA]) bestimmt und mit einer präoperativen Aufnahme, behelfsweise mit der Gegenseite, verglichen. Ein Overstuffing des anterioren Kompartiments oder eine pathologische Gelenkkinematik führt zu einem Vorschub des Femurs und damit zu einer Vergrößerung des PTA mit erhöhter Spannung der Retinakula.

Auch eine relevante Reduktion ( $4 \mathrm{~mm}$ ) des posterioren Offsets oder Verschiebung der femoralen oder tibialen Gelenklinie können ebenfalls, insbesondere bei kreuzbanderhaltenden Implantatdesigns, zu Bewegungseinschränkungen führen $[2,13,16]$.

Eine häufige Ursache von Bewegungseinschränkungen nach Knietotalendoprothese ist die Patella baja. Sie ist vergesellschaftet mit einem erhöhten Patellaanpressdruck und kann zu einem Impingement des Hoffa-Körpers gegen das Inlay führen. Die echte Patalla baja ist selten und Folge einer Schrumpfung des Patellarbandes z. B. nach Bone-tendon-bone-Plastik einer VKB(Vorderes Kreuzband)-Ruptur. Die weitaus häufigere Pseudo-Patella baja ist Folge einer tibialen Gelenklinienverschiebung durch die Totalendoprothese (TEP) oder im Zustand nach aufklappender hoher Tibiaosteotomie.

Impingement durch Implantatüberhang ist vermeidbar und einfach $\mathrm{zu}$ diagnostizieren. Es kann zwischen statischem und dynamischem Impingement unterschieden werden. Das statische Impingement durch Implantatüberhang wird meist tibial posterolateral im Be- reich der Popliteussehne beobachtet, seltener anteromedial im Bereich des oberflächlichen Innenbandkomplexes. Das dynamische Impingement wird insbesondere bei flotierender oder rotierender Plattform und hier bevorzugt bei Größenmismatch zwischen Femur- und Tibiateil (Femur > Tibia) beobachtet.

Auch eine Implantatlockerung kann zu einer schmerzhaften Gelenksteife führen. Die Diagnostik ist nicht trivial, da nur die Implantatmigration beweisend ist. Selbst durchgehende Säume müssen nicht beweisend für eine Lockerung sein, wenngleich sie bis zum Beweis des Gegenteils als Lockerungszeichen gewertet werden sollten. Die Szintigraphie kann in solchen Zweifelsfällen hilfreich sein, wobei ihre Aussagekraft im ersten Jahr nach Implantation beschränkt ist. Sie erlaubt neben der Sicherung einer nativradiologisch vermuteten Implantatlockerung auch die Beurteilung der Patella. Eine sog. „hot patella“ ist ein Zeichen für eine Überbeanspruchung des Patellofemoralgelenks und zeigt gute Ergebnisse nach Retropatellarersatz [1].

\section{Gelenkferne Ursachen}

Somatische gelenkferne Ursachen einer Bewegungsstörung sind Koxarthrose, Spinalkanal- oder Neuroforamenstenose oder eine die Gelenke betreffende neurologische Grunderkrankung (u.a. Morbus Parkinson, Spastik verschiedener Genese, multiple Sklerose). Weitaus häufiger werden jedoch Somatisierungsstörungen beobachtet. Bei entsprechendem Verdacht ist eine entsprechende fachärztliche Abklärung erforderlich.

Eine besondere Herausforderung stellt das Rentenbegehren dar, das in Abhängigkeit der medizinischen Kenntnisse des Patienten nur schwer zu diagnostizieren ist.

\section{Vorgehen}

Das Vorgehen bei Vorliegen einer Gelenksteife nach Knieendoprothese sollte standardisiert erfolgen, um keine mögliche Ursache zu übersehen. Hier ist ein den Gegebenheiten jeder Klinik angepasster diagnostischer Algorithmus sinnvoll. Die Reihenfolge der Untersuchun-
Trauma Berufskrankh $2017 \cdot 19$ (Suppl 3):S272-S274 https://doi.org/10.1007/s10039-017-0303-5

○) Springer Medizin Verlag GmbH 2017

\section{G. Matziolis}

\section{Periprothetische Gelenksteife. Ursachen und Vorgehen}

\section{Zusammenfassung}

Die periprothetische Kniegelenksteife ist nach posttraumatischer Arthrose häufiger als nach primärer Arthrose. Es sind zahlreiche mechanische, biologische und sonstige Risikofaktoren bekannt, die eine periprothetische Gelenksteife begünstigen. Ein standardisierter diagnostischer

Algorithmus soll potenzielle Ursachen identifizieren. Die Therapie richtet sich nach der wahrscheinlichsten Ursache der Gelenksteife.

Schlüsselwörter

Arthrofibrose · Infektion · Malalignment . Instabilität · Diagnostischer Algorithmus

\section{Periprosthetic joint stiffness. Causes and approaches}

\section{Abstract}

Stiffness after total knee arthroplasty is more common after posttraumatic than after primary arthritis of the knee. Numerous mechanical, biological and other risk factors promoting joint stiffness after knee arthroplasty are known. A standardized diagnostic algorithm aims at identifying potential risk factors. The therapy should be based on the most probable identified cause of joint stiffness.

\section{Keywords}

Arthrofibrosis · Infection - Malalignment . Instability - Diagnostic algorithm

gen sollte sich dabei nach Invasivität der Diagnostik und Wahrscheinlichkeit der vermuteten Ursache richten.

Nach Identifikation einer (oder mehrerer) potenzieller Ursachen einer $\mathrm{Ge}$ lenksteife sollte die Therapie so kausal wie möglich erfolgen [21].

Sollte sich kein Risikofaktor für eine Gelenksteife identifizieren lassen, ist die symptomatische Therapie mittels „brisement force" bis zur 6 . Woche sicher, bis zur 12. Woche mit erhöhter Komplikationsrate (Fraktur, Patellarsehnenruptur) indiziert $[14,29]$. Danach wird eine arthroskopische Arthrolyse empfohlen, da 
sie zu besseren Langzeitergebnisse bei geringerer Invasivität als die offene Arthrolyse führt [8].

Kommt es zum Rezidiv einer Bewegungseinschränkung ist eine primäre Arthrofibrose wahrscheinlich. Wiederholte Arthrolysen sind dann nicht Erfolg versprechend. Wenngleich es noch keine allgemein akzeptierten Therapiestandards gibt, scheint eine Schmerzen vermeidende sanfte multimodale Therapie Erfolg versprechend [7].

\section{Fazit für die Praxis}

- Die periprothetische Gelenksteife nach posttraumatischer Arthrose ist häufiger als nach primärer Arthrose.

- Es gibt zahlreiche unterschiedliche Ursachen.

- Eine Infektion sollte stets ausgeschlossen werden.

- Ein der jeweiligen Einrichtung angepasster diagnostischer Algorithmus ist hilfreich.

- Die Therapie richtet sich nach der wahrscheinlichsten Ursache.

- Kann keine Ursache identifiziert werden, kommen "brisement force", arthroskopische oder offene Arthrolyse infrage.

- Die primäre Arthrofibrose ist eine Ausschlussdiagnose und sollte nicht wiederholt operativ angegangen werden.

\section{Korrespondenzadresse}

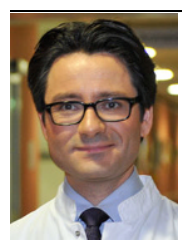

Prof. Dr. G. Matziolis

Campus Eisenberg,

Orthopädische Klinik des

Universitätsklinikums Jena

Klosterlausnitzer Str. 81,

07607 Eisenberg,

Deutschland

g.matziolis@krankenhauseisenberg.de

\section{Einhaltung ethischer Richtlinien}

Interessenkonflikt. G. Matziolis gibt an, dass kein Interessenkonflikt besteht.

Dieser Beitrag beinhaltet keine vom Autor durchgeführten Studien an Menschen oder Tieren.

The supplement containing this article is not sponsored by industry.

\section{Literatur}

1. Ahmad R, Kumar GS, Katam K et al (2009) Significance of a "hot patella" in total knee replacement without primary patellar resurfacing. Knee 16:337-340. https://doi.org/10.1016/j.knee. 2009.02.001

2. Bellemans J, Banks S, Victor J et al (2002) Fluoroscopic analysis of the kinematics of deep flexion in total knee arthroplasty. Influence of posterior condylar offset. J Bone Joint Surg Br 84:50-53

3. Boldt JG, Munzinger UK, Zanetti M, Hodler J (2004) Arthrofibrosis associated with total knee arthroplasty: gray-scale and power Doppler sonographic findings. AJR Am J Roentgenol 182:337-340. https://doi.org/10.2214/ajr.182.2. 1820337

4. Chambers AW, Wood AR, Kosmopoulos V et al (2016) Effect of posterior tibial slope on flexion and anterior-posterior tibial translation in posterior cruciate-retaining total knee arthroplasty. J Arthroplasty 31:103-106. https://doi.org/10.1016/j. arth.2015.08.027

5. Christensen CP, Crawford JJ, Olin MD, Vail TP (2002) Revision of the stiff total knee arthroplasty. JArthroplasty 17:409-415

6. Claassen L, Ettinger S, Pastor M-F et al (2016) The value of arthroscopic neosynovium biopsies to diagnose periprosthetic knee joint lowgrade infection. Arch Orthop Trauma Surg 136:1753-1759. https://doi.org/10.1007/s00402016-2574-X

7. Faust I, Traut $P$, Nolting $F$ et al (2015) Human xylosyltransferases-mediators of arthrofibrosis? New pathomechanistic insights into arthrofibrotic remodeling after knee replacement therapy. Sci Rep 5:12537.https://doi.org/10.1038/srep12537

8. Fitzsimmons SE, Vazquez EA, Bronson MJ (2010) How to treat the stiff total knee arthroplasty?: a systematic review. Clin Orthop Relat Res 468:1096-1106. https://doi.org/10.1007/s11999010-1230-y

9. Gandhi R, de Beer J, Leone J et al (2006) Predictive riskfactors for stiff knees in total knee arthroplasty. J Arthroplasty 21:46-52. https://doi.org/10.1016/ j.arth.2005.06.004

10. Geller JA, Lakra A, Murtaugh T (2017) The use of electronic sensor device to augment ligament balancing leads to a lower rate of arthrofibrosis after total knee arthroplasty. J Arthroplasty 32:1502-1504. https://doi.org/10.1016/j.arth. 2016.12.019

11. Harvie P, Larkin J, Scaddan M et al (2013) Stiffness after total knee arthroplasty: does component alignment differ in knees requiring manipulation? A retrospective cohort study of 281 patients. J Arthroplasty 28:14-19. https://doi.org/10.1016/ j.arth.2012.03.003

12. Higuera CA, Zmistowski B, Malcom T et al (2017) Synovial fluid cell count for diagnosis of chronic periprosthetic hip infection. J Bone Joint Surg Am 99:753-759. https://doi.org/10.2106/JBJS.16. 00123

13. Hofmann AA, Kurtin SM, Lyons Setal (2006) Clinical and radiographic analysis of accurate restoration of the joint line in revision total knee arthroplasty. J Arthroplasty 21:1154-1162. https://doi.org/10. 1016/j.arth.2005.10.026

14. Issa K, Kapadia BH, Kester M et al (2014) Clinical, objective, and functional outcomes of manipulation under anesthesia to treat knee stiffness following total knee arthroplasty. J Arthroplasty 29:548-552. https://doi.org/10. 1016/j.arth.2013.07.046
15. Janz V, Wassilew Gl, Hasart O et al (2013) Evaluation of sonicate fluid cultures in comparison to histological analysis of the periprosthetic membrane for the detection of periprostheticjoint infection. Int Orthop 37:931-936. https://doi.org/ 10.1007/s00264-013-1853-1

16. Kazemi SM, Daftari Besheli L, Eajazi A et al (2011) Pseudo-patella baja after total knee arthroplasty. Med Sci Monit 17:CR292-6

17. Kim J,Nelson CL, LotkePA (2004)Stiffness aftertotal knee arthroplasty. Prevalence of the complication and outcomes of revision. J Bone Joint Surg Am 86A:1479-1484

18. Krenn V, Morawietz L, Kienapfel H et al (2013) Revised consensus classification. Histopathological classification of diseases associated with joint endoprostheses. Z Rheumatol 72:383-392. https://doi.org/10.1007/s00393-012-1099-0

19. Lachiewicz PF, Watters TS, Jacobs JJ (2016) Metal hypersensitivity and total knee arthroplasty. J Am Acad Orthop Surg 24:106-112. https://doi.org/10. 5435/JAAOS-D-14-00290

20. Matziolis G, Adam J, Perka C (2010) Varus malalignmenthasnoinfluence on clinical outcome in midterm follow-up after total knee replacement Arch Orthop Trauma Surg 130:1487-1491. https:// doi.org/10.1007/s00402-010-1064-9

21. Moya-Angeler J, Bas MA, Cooper HJ et al (2017) Revision arthroplasty for the management of stiffness after primary TKA. J Arthroplasty 32:1935-1939. https://doi.org/10.1016/j.arth. 2017.01.010

22. Murphy MT, Skinner TL, Cresswell AG et al (2014) The effect of knee flexion contracture following total knee arthroplasty on the energy cost of walking. J Arthroplasty 29:85-89. https://doi.org/ 10.1016/j.arth.2013.04.039

23. Perry J, Antonelli D, Ford W (1975) Analysis of kneejoint forces during flexed-knee stance. J Bone Joint Surg Am 57:961-967

24. Schäfer P, Fink B, Sandow D et al (2008) Prolonged bacterial culture to identify late periprosthetic joint infection: a promising strategy. Clin Infect Dis 47:1403-1409. https://doi.org/10.1086/592973

25. Shahi A, Parvizi J (2016) The role of biomarkers in the diagnosis of periprosthetic joint infection. EFORT Open Rev 1:275-278. https://doi.org/10. 1302/2058-5241.1.160019

26. Tani S, Lepetsos P, Stylianakis A et al (2017) Superiority of the sonication method against conventional periprosthetic tissue cultures for diagnosis of prosthetic joint infections. Eur J Orthop Surg Traumatol. https://doi.org/10.1007/ s00590-017-2012-y

27. Thomas $P$, von der Helm C, Schopf C et al (2015) Patients with intolerance reactions to total knee replacement: combined assessment of allergy diagnostics, periprosthetic histology, and periimplant cytokine expression pattern. Biomed Res Int 2015:910156. https://doi.org/10.1155/2015/ 910156

28. Trampuz A, Hanssen AD, Osmon DR et al (2004) Synovial fluid leukocyte count and differential for the diagnosis of prosthetic knee infection. Am J Med 117:556-562. https://doi.org/10.1016/j. amjmed.2004.06.022

29. Vanlommel L, Luyckx T, Vercruysse G et al (2016) Predictors of outcome after manipulation under anaesthesia in patients with a stiff total knee arthroplasty. Knee Surg Sports Traumatol Arthrosc https://doi.org/10.1007/s00167-016-4413-6

30. Wu L-D, Xiong Y, Yan S-G, Yang Q-S (2005) Total knee replacement for posttraumatic degenerative arthritis of the knee. Chin J Traumatol 8:195-199 